\title{
The Impact of Age on Survival in CLL Patients Receiving Ibrutinib as Initial Therapy
}

This article was published in the following Dove Press journal:

Blood and Lymphatic Cancer: Targets and Therapy

\begin{abstract}
Chaitra Ujjani, ${ }^{1}$ Anthony Mato, ${ }^{2}$ Brian T Hill, ${ }^{3}$ John N Allan, (D) ${ }^{4}$ Frederick Lansigan, (D) ${ }^{5}$ Ryan Jacobs, ${ }^{6}$ Alan Skarbnik, ${ }^{7}$ Hande Tuncer, ${ }^{8}$ John Pagel, ${ }^{9}$ Danielle Brander, ${ }^{10}$ Bruce Cheson, (iD) ${ }^{11}$ Paul Barr, ${ }^{12}$ Lindsey E Roeker, ${ }^{2}$ Jeffrey Pu, (iD ${ }^{13}$ Nirav N Shah, ${ }^{14}$ Andre Goy, iD ${ }^{15}$ Stephen J Schuster, ${ }^{16}$ Nicole Lamanna, ${ }^{17}$ Alison Sehgal, ${ }^{18}$ Constantine S Tam, ${ }^{19}$ Mazyar Shadman'

'Seattle Cancer Care Alliance, Fred Hutchinson Cancer Research Center, Seattle, WA, USA; ${ }^{2}$ Division of Hematological Oncology, Memorial Sloan Kettering Cancer Center, New York, NY, USA; ${ }^{3}$ Taussig Cancer Institute, Cleveland Clinic, Cleveland, OH, USA; ${ }^{4}$ Division of Hematology and Medical Oncology, New York Presbyterian \& Weill Cornell, New York, NY, USA; ${ }^{5}$ Norris Cotton Cancer Center, Dartmouth-Hitchcock Medical Center, Lebanon, NH, USA; ${ }^{6}$ Department of Hematologic Oncology and Blood Disorders, Levine Cancer Institute, Charlotte, NC, USA; ${ }^{7}$ Novant Health Cancer Institute, Charlotte, NC 28204, USA; ${ }^{8}$ Lowell General Hospital, Tufts Medical Center, Boston, MA, USA; ${ }^{9}$ Center for Blood Disorders and Stem Cell Transplantation, Swedish Cancer Institute, Seattle, WA, USA; ${ }^{10}$ Division of Hematologic Malignancies and Cellular Therapy, Duke University, Durham, NC, USA; "'Lombardi Comprehensive Cancer Center, Georgetown University Hospital, Washington, DC, USA; ' ${ }^{2}$ Wilmot Cancer Institute, University of Rochester Medical Center, Rochester, NY, USA; ${ }^{3}$ Division of Hematology/Oncology, SUNY Upstate Medical University, Syracuse, NY, USA; ${ }^{14}$ Division of Hematology \& Oncology, Medical College of Wisconsin, Milwaukee, WI, USA; ${ }^{15}$ John Theurer Cancer Center, Hackensack University Medical Center, Hackensack, NJ, USA; ${ }^{16}$ Abramson Cancer Center, University of Pennsylvania, Philadelphia, PA, USA; ${ }^{17}$ Herbert Irving Comprehensive Cancer Center, Columbia University Medical Center, New York, NY, USA;

${ }^{18}$ University of Pittsburgh Medical Center, Pittsburgh, PA, USA; ${ }^{19}$ Peter McCallum Cancer Centre, University of Melbourne, East Melbourne, $\mathrm{VI}$, Australia
\end{abstract}

Correspondence: Chaitra Ujjani

Seattle Cancer Care Alliance, Fred Hutchinson Cancer Research Center, 825 Eastlake Ave E, Mail Stop CE3-300, Seattle, WA, USA

Tel + I 206-606-1955

Fax + I 206-606-II30

Email ujjani@uw.edu
Introduction: Recent randomized trials have demonstrated the efficacy of ibrutinib-based therapy in the treatment of patients with CLL. In Alliance A041202, a higher than expected number of unexplained deaths were reported with front-line ibrutinib in a patient population aged at least 65 years compared to ECOG 1912, which included patients up to 70 years of age. Methods: Therefore, we conducted a retrospective analysis to investigate whether ibrutinib was associated with a greater mortality in older patients outside of a clinical trial setting. This multicenter analysis was performed by investigators at 20 academic and community practices.

Results: Amongst the 391 patients included, there was no correlation between age and response rate, PFS, or OS. However, there was a trend to higher rate of deaths in patients $>65$ years-old ( $8.7 \%$ vs $3.8 \%, p=0.097)$, with an increased number of early deaths ( 13 vs $4, p=0.3$ ). Conclusion: These data suggest greater intolerance, and possibly mortality, with ibrutinib in an older population. Patients should be educated regarding the potential complications related to ibrutinib and symptoms of concern to report.

Keywords: CLL, ibrutinib, elderly

\section{Introduction}

Recently published cooperative group studies, Alliance A041202 and ECOG E1912, have established the role of ibrutinib over chemoimmunotherapy for the front-line treatment of chronic lymphocytic leukemia (CLL). ${ }^{1,2}$ Notably, Alliance A041202, which evaluated patients $\geq 65$-years-old receiving ibrutinib with or without rituximab or the combination of bendamustine and rituximab (BR), reported a higher than expected number of deaths in both of the ibrutinib-containing arms. ${ }^{1}$ Ten unexplained/unknown deaths occurred among the 361 patients receiving ibrutinib; whereas 1 such event occurred in the BR arm $(n=176)$. These data were in contrast to ECOG E1912 in which ibrutinib plus rituximab was associated with an improvement in overall survival (OS) compared to FCR (fludarabine, cyclophosphamide, rituximab) in patients less than 70 years of age. ${ }^{2}$ Together, these data suggest ibrutinib may be associated with significant adverse events (AE) and greater mortality in an elderly CLL population. We sought to investigate this hypothesis in previously untreated patients who received commercially available ibrutinib as initial therapy for CLL.

\section{Methods}

This multicenter retrospective analysis was performed by investigators at 20 academic and community practices. The study was approved by the institutional review board at 
each participating institution (Supplementary Materials). Informed consent was waived because the research involved no more than minimal risk to the patient. In compliance with the Declaration of Helsinki, patient confidentiality was maintained through de-identified data collection practices. Patients were considered eligible for inclusion if they received ibrutinib for the front-line treatment of CLL through commercial sources. Medical chart review via diagnostic codes was performed to identify all CLL patients at each institution. Investigators utilized chart review, electronic medical records, and related databases to obtain required information. Patient demographics, prognostic markers, ibrutinib dosing administration, and clinical outcomes data were collected. The International Workshop on Chronic Lymphocytic Leukemia (iwCLL, 2008) criteria were used to define response and progression of disease. ${ }^{3}$

The primary endpoint was OS as determined by the Kaplan Meier method. ${ }^{4}$ OS was defined as time from initiation of ibrutinib to death. Comparisons of survival were made using COX regression analyses. ${ }^{5,6}$ All other comparison analyses were descriptive. Early deaths were defined as a death from any cause occurring within 12 months of ibrutinib treatment initiation. Secondary endpoints included response rates, progression-free survival (PFS), discontinuation rates, and reasons for discontinuation. PFS was defined as time from ibrutinib initiation to progression or death from any cause. Patients were otherwise censored at the time of last follow-up. Statistical analyses were performed using STATA 10.1 (Stata Statistical Software: Release 10, 2007; StataCorp LP, College Station, TX). All tests were two-sided at the $5 \%$ level.

\section{Results}

Of the 391 patients identified, 59\% were 65 years or older ( $\geq 65 \mathrm{YO}$ ). The median age at initiation of ibrutinib was 63 years (range 36-96). Patient characteristics are detailed in Table 1. Poor risk prognostic features were similar in patients stratified by age at start of ibrutinib $(\geq 65 \mathrm{YO}$ and $<65$ YO cohorts, respectively): del $17 p(29 \%, 30 \%)$, TP53 mutation $(21 \%, 19 \%)$, IGHV unmutated (71\%, $64 \%)$, and complex karyotype (25\%, 21\%).

The median time from diagnosis to initiation of ibrutinib was 35 months for patients $\geq 65 \mathrm{YO}$ and 25 months for those $<65$ YO. Eleven percent of older patients were initiated on a lower than standard dose of ibrutinib (420 mg daily) in contrast to $2 \%$ of younger patients ( $\mathrm{p}=0.02$ ). In older patients, $20 \%$ required dose reduction compared to $13 \%$ of younger patients $(\mathrm{p}=0.07$ ). Dose interruptions occurred in $46 \%$ of older
Table I Patient Characteristics

\begin{tabular}{|c|l|l|}
\hline & $<65$ YO (n=160) & $\geq \mathbf{6 5}$ YO (n=23 I) \\
\hline $\begin{array}{c}\text { Sex } \\
\text { M }\end{array}$ & $63 \%$ & \\
F & $37 \%$ & $62 \%$ \\
\hline Race & & $38 \%$ \\
Caucasian & $90 \%$ & \\
Other & $10 \%$ & $94 \%$ \\
\hline del I7p & $(n=158)$ & $6 \%$ \\
Absent & $70 \%$ & $(n=211)$ \\
Present & $30 \%$ & $71 \%$ \\
\hline TP53 mutation & $(n=102)$ & $29 \%$ \\
Absent & $81 \%$ & $(n=117)$ \\
Present & $19 \%$ & $79 \%$ \\
\hline del I Iq & $(n=158)$ & $21 \%$ \\
Absent & $79 \%$ & $(n=209)$ \\
Present & $21 \%$ & $86 \%$ \\
\hline Complex Karyotype & $(n=122)$ & $14 \%$ \\
Absent & $79 \%$ & $(n=157)$ \\
Present & $21 \%$ & $75 \%$ \\
\hline IgVH & $(n=102)$ & $25 \%$ \\
Unmutated & $64 \%$ & $(n=119)$ \\
Mutated & $36 \%$ & $71 \%$ \\
\hline
\end{tabular}

and $36 \%$ of younger patients $(\mathrm{p}=0.04)$; the median duration of dose interruption was 13 and 10 days, respectively $(\mathrm{p}=0.49)$. Ibrutinib discontinuation occurred in similar frequency for older and younger patients $(25 \%, 22 \%)$. Intolerance was the most common reason for discontinuation in both age groups ( $\mathrm{n}=36$ and 20, respectively), followed by CLL progression ( $\mathrm{n}=$ $6,6)$, and Richter's transformation $(\mathrm{n}=5,4)$.

Investigator assessed overall and complete response rates for ibrutinib were $79 \%$ and $15 \%$ in patients $\geq 65 \mathrm{YO}$ and $85 \%$ and $20 \%$ in those $<65$ YO. There was no difference in PFS based on age (HR 1.13, p =0.69), Figure 1. At a median follow up of 13.8 months (range 1-76 months), a total of 26 deaths were observed. There was a higher proportion of deaths in the older cohort ( $8.7 \%$ vs $3.8 \%, 20$ deaths vs 6 deaths), though difference in OS did not reach statistical significance (HR 2.1, $\mathrm{p}=0.097$ ). Figure 1 . The number of early deaths, defined as occurring within the first 12 months of therapy, was 13 in patients $\geq 65$ YO compared to 4 in patients $<65 \mathrm{YO}, \mathrm{p}=0.3$.

\section{Conclusion}

In this cohort of nearly 400 patients who received single agent ibrutinib for the front-line treatment of CLL, age did 

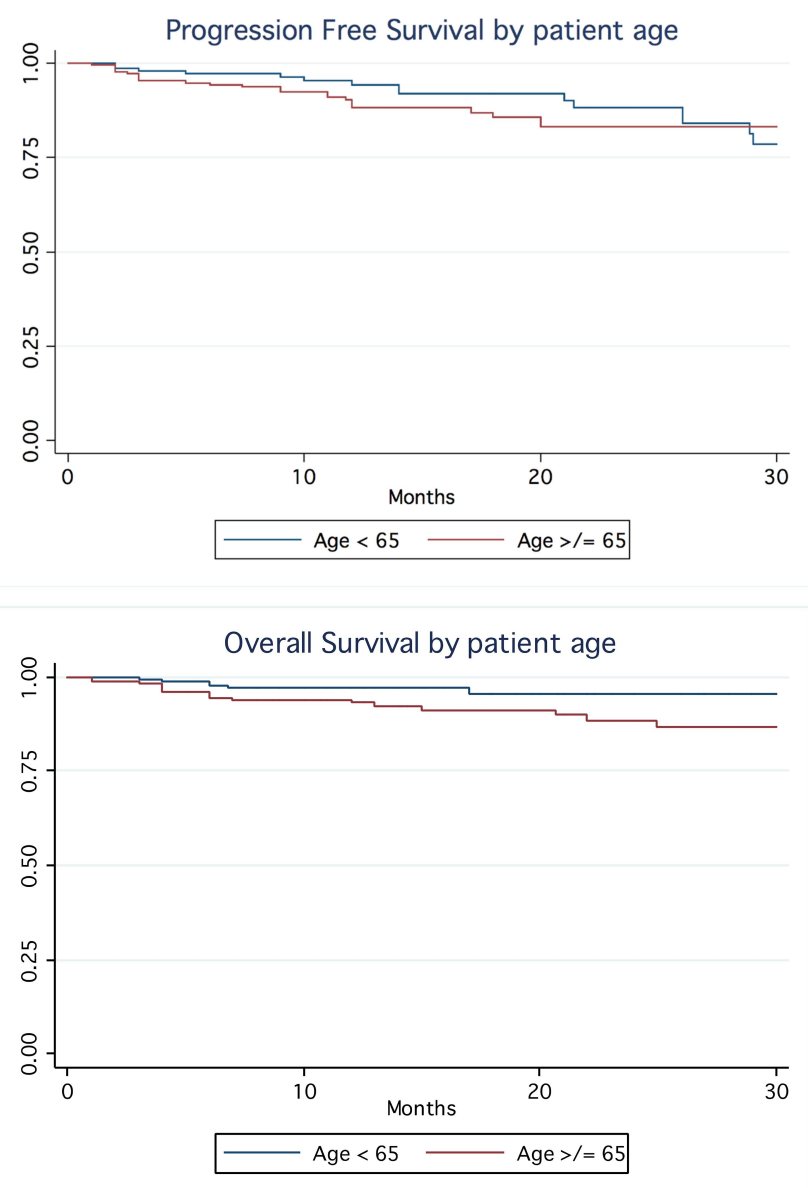

Figure I Survival by age.

not correlate with response rates, progression-free survival, or overall survival. Nevertheless, a greater than twofold increase in death was noted in patients $\geq 65$-years-old compared to the younger cohort. Similar to the data reported in the Alliance A041202 study, where the majority of unexplained deaths with ibrutinib occurred within the first two years of treatment, early deaths in our analysis appeared to occur more frequently in the older patient population. ${ }^{1}$ These data support our initial hypothesis that ibrutinib may be associated with a greater early mortality in an older CLL population.

In this analysis, nearly $50 \%$ of the older population required dose interruptions and $20 \%$ required dose reductions suggesting issues with drug tolerability related to AEs. Furthermore, it appears that practitioners anticipated an increase in AEs in the older population as they more frequently prescribed lower than standard doses of ibrutinib at initiation of therapy. Alternatively, this may represent decreased experience with the drug in the early days of approval. Despite these findings, the incidence of ibrutinib discontinuation was relatively comparable between the different age groups. Additionally, intolerance to ibrutinib remained the most common reason for discontinuation of therapy in all patients.

When comparing the grade 3-5 adverse events that occurred with ibrutinib in the older Alliance A041202 and younger ECOG E1912 populations, the most notable differences are the increased rates of cardiovascular toxicity, specifically atrial fibrillation ( $9 \%$ vs $3 \%$ ) and hypertension $(29 \% \text { vs } 7 \%)^{1,2}$ A recent retrospective analysis of 562 patients receiving ibrutinib at the Ohio State University also noted a marked incidence of new hypertension of $72 \%$. $^{7}$ Major cardiac events were noted in $17 \%$ of patients and occurred more frequently in those with new or worsening hypertension (19\%). In a separate aggregated data set of over 1000 patients receiving ibrutinib, 10 events of sudden death or cardiac arrest were identified. ${ }^{8}$ The HELIOS study of BR with or without ibrutinib was included in this analysis; 7 events of $\geq$ grade 3 ventricular arrhythmia, cardiac arrests, and sudden deaths were found in the ibrutinib-containing arm compared to 0 with $\mathrm{BR}$ alone. ${ }^{9}$ The higher incidence of these toxicities raises suspicion for a cardiac event as a potential etiology for the unexplained, early deaths noted in both Alliance A041202 and this analysis; however, investigators were unable to confirm this impression. It is also noted that early death is not necessarily the same as sudden death. Unfortunately, a critical limitation of our analysis is that the individual causes of death were not captured uniformly as part of this retrospective data review, nor were the past cardiac histories. We were unable to identify which, if any, adverse events occurred in patients who experienced an early death or assign attribution to drug. However, given the marked efficacy of ibrutinib and the ability of patients to be effectively salvaged after progression, one may assume that toxicity may be a key contributing factor.

Given the quickly evolving treatment landscape for CLL, one of the more complicated decisions oncologists will face is determining the most appropriate novel therapeutic for their individual patient. Ibrutinib is certainly a highly effective therapy for patients of all ages, ensuring a lengthy PFS and OS in elderly patients with high-risk disease. However, as the majority of patients with CLL are elderly and many have comorbidities, ensuring tolerability is crucial. While our data are hypothesis generating only, these findings in combination with those of Woyach et al suggest close monitoring of elderly patients treated with ibrutinib is warranted for toxicity, particularly in the first 
12 months of therapy. ${ }^{1}$ In addition, patients should be educated regarding the potential complications related to treatment and symptoms of concern to report.

Fortunately, a number of effective agents have or will become available to patients in the near future. The CLL-14 study of venetoclax and obinutuzumab recently secured the B-cell lymphoma (BCL)-2 inhibitor a role in the front-line setting. ${ }^{10}$ The safety of venetoclax in an elderly population with comorbidities, including decreased renal function, was demonstrated in this study. However, 5 fatal adverse events were noted during treatment, primarily due to infection, as well as 4 fatal infection events which occurred after completion of venetoclax. The incidence of severe neutropenia was $53 \%$ in this study, highlighting the importance in supporting and monitoring patients appropriately both during and after therapy. Additionally, there are second-generation Bruton tyrosine kinase (BTK) inhibitors in development, including acalabrutinib and zanubrutinib, which may be associated with less atrial fibrillation. $^{11,12}$ Their relative risk of early deaths will be forthcoming with the results of Phase III trials. The combination of a BTK inhibitor and a BCL-2 inhibitor has also demonstrated remarkable efficacy with a shorter required treatment duration and thus less time for drug exposure. ${ }^{13}$ Whether fixed duration combination strategies will result in less toxicity or early deaths is unknown. Moving forward, although the correlation between age and survival was not shown to be statistically significant, we do feel a greater effort must be focused on understanding whether the cause of early mortality in older patients receiving ibrutinib is related to the drug or the patient; additionally, how best to mitigate this problem which may limit the use of this highly effective agent.

\section{Acknowledgments}

This abstract of this paper was presented at the 2019 International Workshop in CLL as a poster presentation with interim findings. https://www.iwcll2019.org/wpcontent/uploads/2019/09/iwCLL-Abstract-Titles.pdf

\section{Author Contributions}

All authors made a significant contribution to the work reported, whether that is in the conception, study design, execution, acquisition of data, analysis and interpretation, or in all these areas; took part in drafting, revising or critically reviewing the article; gave final approval of the version to be published; have agreed on the journal to which the article has been submitted; and agree to be accountable for all aspects of the work.

\section{Funding}

There was no funding for this project.

\section{Disclosure}

Chaitra Ujjani reports non-financial supports from Pharmacyclics, Abbvie, Genentech, AstraZeneca, and Verastem. Anthony Mato reports grants and personal fees from TG Therapeutics, Celgene, Abbvie, Loxo, Sunesis, Genentech, DTRM, AstraZeneca, Octopharma, Janssen, Regeneron, Adaptive, and Pharmacyclics. Brian T Hill reports grants, personal fees from Pharmacyclics, Abbvie, AstraZeneca. John N Allan reports personal fees from Abbvie, Janssen, Pharmacyclics, Genentech, TG Therapeutics, Verastem, Sunesis, and Ascentage Pharma Group. Frederick Lansigan reports consulting fees from Acrotech and is part of the steering committee for Celgene. Alan Skarbnik is a consultant for Janssen, Pharmacyclics, Abbvie, Celgene, Kite, Alexion, Jazz Pharmaceuticals, and AstraZeneca and received payments for lectures including service on speakers bureaus from Janssen, Pharmacyclics, Abbvie, Celgene, Kite, Alexion, Jazz Pharmaceuticals, AstraZeneca, Beigene, Seattle Genetics, Genentech, and Verastem. Ryan Jacobs reports grants and/or personal fees from Pharmacyclics, Genentech, Abbvie, Verastem, TG Therapeutics, Janssen, Astra Zeneca. John Pagel reports personal fees from Gilead, Beigene, Loxo, and AstraZeneca. Danielle Brander reports grants, non-financial support from AbbVie, ArQule, Ascentage, Astra Zeneca, BeiGene, DTRM, Genentech, Juno/Celgene/BMS, MEI Pharma, Pharmacyclics, Pfizer, Teva, TG Therapeutics, Tolero, Verastem, and memberships with other guidelines/registry memberships (when sponsored or consultant also included under sponsor mentioned): NCCN panel member, informCLL registry steering committee (AbbVie), REAL registry steering committee (Verastem), and Biosimilars outcomes research panel (Pfizer). Bruce Cheson reports personal fees from Abbvie, Pharmacyclics, Celgene, TG Therapeutics, Beigene, AstraZeneca, and Genentech. Paul Barr is consultant for Abbvie, Pharmacyclics, Gilead, Genentech, TG therapeutics, Seattle Genetics, Celgene, Morphosys, Verastem, AstraZeneca, and Janssen. Lindsey Roeker reports family member with minority ownership interest in AbbVie and Abbott Laboratories. Andre Goy reports personal fees from Janssen, Pharmacyclics, during the conduct of the study; as principal investigator for and received funding 
for institution from Genentech-Hoffman La Roche, including personal fees from Acerta, AstraZeneca, Celgene, Constellation, Infinity, Infinity Verastem, Karyopharm, Kite pharma, Elsevier's PracticeUpdate Oncology, Gilead, Medscape, MJH Associates, OncLive Peer Exchange, Physcians Education Resource, LLC, Xcenda, and COTA, outside the submitted work. Stephen J Schuster reports personal fees and/or grants from AstraZeneca, Merck, Novartis, Juno/Celgene, Genentech/Roche, Loxo Oncology, Tessa Therapeutics, AlloGene, BeiGene, and Celgene. Dr Nicole Lamanna received research support to institution and advisory board honorarium from Abbvie, Astra Zeneca, Beigene, Genentech, Gilead, Juno, Janssen, Mingsight, Pharmacyclics, TG Therapeutics, Celgene, and Oncternal. Constantine Tam reports personal fees from Abbvie, Janssen, Pharmacyclics. Mazyar Shadman reports personal fees from Abbvie, Genentech, Astra Zeneca, Sound Biologics, Pharmacyclics, Verastem, ADC therapeutics, Beigene, Cellectar, BMS, Mophosys and Atara Biotherapeutics, grants from Mustang Bio, Celgene, Pharmacyclics, Gilead, Genentech, Abbvie, TG therapeutics, Beigene, Astra Zeneca, Sunesis, and Beigene. The authors report no other conflicts of interest in this work.

\section{References}

1. Woyach JA, Ruppert AS, Heerema N, et al. Ibrutinib regimens versus chemoimmunotherapy in older patients with untreated CLL. $N$ Engl $J$ Med. 2018;379(26):2517-2528. doi:10.1056/NEJMoa1812836

2. Shanafelt TD, Wang XV, Kay NE, et al. Ibrutinib-rituximab or chemoimmunotherapy for chronic lymphocytic leukemia. $N$ Engl J Med. 2019;381(5):432-443. doi:10.1056/NEJMoa1817073
3. Hallek M, Cheson BD, Catovsky D, et al. Guidelines for the diagnosis and treatment of chronic lymphocytic leukemia: a report from the international workshop on chronic lymphocytic leukemia updating the National Cancer Institute-working group 1996 guidelines. Blood. 2008;111(12):5446-5456. doi:10.1182/blood-2007-06-093906

4. Kaplan EL, Meier P. Nonparametric estimation from incomplete observations. J Am Stat Assoc. 1958;53(282):457-481. doi:10.1080/ 01621459.1958 .10501452

5. Anderson P, Gill R. Cox's regression model for counting processes: a large sample study. Ann Statist. 1982;10(4):1100-1120. doi:10. 1214/aos/1176345976

6. Cox DR. Analysis of Survival Data. Routledge; 2018.

7. Dickerson T, Wiczer T, Waller A, et al. Hypertension and incident cardiovascular events following ibrutinib initiation. Blood. 2019;134 (22):1919-1928. doi:10.1182/blood.2019000840

8. Lampson B, Yu L, Glynn R, et al. Ventricular arrhythmias and sudden death in patients taking ibrutinib. Blood. 2017;129(18):2581-2584. doi:10.1182/blood-2016-10-742437

9. Chanan-Khan A, Cramer P, Demirkan F, et al. Ibrutinib combined with bendamustine and rituximab compared with placebo, bendamustine, and rituximab for previously treated chronic lymphocytic leukaemia or small lymphocytic lymphoma (HELIOS): a randomised, double-blind, Phase 3 study. Lancet Oncol. 2016;17(2):200-211. doi:10.1016/S1470-2045(15)00465-9

10. Fischer K, Al-Sawaf O, Bahlo J, et al. Venetoclax and obinutuzumab in patients with CLL and coexisting conditions. $N$ Engl J Med. 2019;380(23):2225-2236. doi:10.1056/NEJMoa1815281

11. Awan FT, Schuh A, Brown JR, et al. Acalabrutinib monotherapy in patients with chronic lymphocytic leukemia who are intolerant to ibrutinib. Blood Adv. 2019;3(9):1553-1562. doi:10.1182/bloodadvances. 2018030007

12. Tam C, Trotman J, Opat S, et al. Phase 1 study of the selective BTK inhibitor zanubrutinib in B-cell malignancies and safety and efficacy evaluation in CLL. Blood. 2019;134(11):851-859. doi:10.1182/ blood.2019001160

13. Wierda W, Siddiqi T, Flinn I, et al. Phase 2 CAPTIVATE results of ibrutinib plus venetoclax in first-line chronic lymphocytic leukemia. $J$ Clin Oncol. 2018;36(15_suppl):Abstract 7502. doi:10.1200/ JCO.2018.36.15_suppl.7502

\section{Publish your work in this journal}

Blood and Lymphatic Cancer: Targets and Therapy is an international, peer-reviewed, open access journal focusing on blood and lymphatic cancer research, identification of therapeutic targets and the optimal use of preventative and integrated treatment interventions to achieve improved outcomes, enhanced survival and quality of life for the cancer patient. The manuscript management system is completely online and includes a very quick and fair peer-review system. Visit http://www.dovepress.com/testimonials.php to read real quotes from published authors. 